\title{
An Improved Formulation of Some Theorems and Axioms in the Axiomatic Foundation of the Hilbert Space Structure of Quantum Mechanics
}

\author{
G. LUDWIG \\ Institut für Theoretische Physik (I) der Universität Marburg
}

Received September 15, 1971

\begin{abstract}
In Ref. [1] the axiomatic foundation of the Hilbert space structure of quantum mechanics was outlined. Apart from a set of physically plausible axioms, the (mathematical) assumption (V 1) of the minimal-decomposition property of the basenorm space $B$ was incorporated into the axiomatic scheme of the theory.

It is shown in the present paper that the assumption (V 1) is superfluous. In the first part of the paper we give a short summary of the axioms; in the second part the main theorems are proved without using assumption (V 1).
\end{abstract}

\section{Introduction}

In [1] a set of axioms for quantum mechanics were motivated by formulating the principal laws of measurement; from these axioms it is possible to deduce the Hilbert space structure of quantum mechanics. In [2] we have attempted to give a more detailed physical interpretation of one of these axioms. A survey of the conceptual structure of our axiomatic scheme is given in [3]. In the present paper we will propose an improved formulation of some of the axioms and theorems of [1]. The concepts and symbols as defined in [1] (Chapter III) are used throughout.

In Ref. [4] (\$3.3) the mapping principle 3c (Abbildungsprinzip 3c) of [1], Chapter III, $\S 1.5$, has been reformulated; it seemed more suitable to take, for the elements of $\underline{\underline{K}}$, more general subsets of $\tilde{K}$ than those given by the mapping principle $3 \mathrm{c}$. As explained in $[4], \S 3.3$, this may be done if the mapping principle $3 \mathrm{c}$ is replaced by a new set of postulates introducing a set $\phi$ of "selection-procedures" (Auswahlverfahren) (cf. Ref. [4], $\S 3.3$ ). The mapping principle $3 \mathrm{~d}$ (Abbildungsprinzip 3d) of Ref. [1], Chapter III, $\S 1.5$, can also be replaced by selection-procedures in a quite 
analogous way. Therefore it is not necessary to repeate here the argument in detail.

The main improvement in the axiomatic formulation of quantum mechanics achieved by replacing the mapping principles $3 \mathrm{c}$ and $3 \mathrm{~d}$ by the selection-procedures was to obtain a clearer insight into the physical meaning of the convex combinations of "ensembles" and "effects", i.e. a more comprehensive formulation of the physical interpretation of the elements of $K$ and $\hat{L}$. Using the concepts introduced in Ref. [4], $\S 3.3$, it is no longer necessary to give any further physical interpretation of the convex combinations $\sum_{i} \lambda_{i} V_{i}$ such as presented in [1], p. 231. In the same way, by replacing the mapping principle $3 \mathrm{~d}$ by a set of selection-procedures, we make any further physical interpretation of the elements of $\hat{L}$ superfluous (such as given in Ref. [1], p. 232).

\section{A Survey of the Axioms}

To start with, we give a short summary of the axioms introduced in Chapter III of Ref. [1]. The fundamental objects of our axiomatic scheme are the sets $\underline{\underline{K}}$ and $\underline{\underline{L}}$. These sets were defined by the selection procedures mentioned above [4]. By $\underline{\underline{M}}^{\prime}$ we denote (as in the mapping principle $3^{\prime}$ of Ref. [1], Chapter III, $\S 1.5$ ) the "set of all experiments" consisting of one "preparing-part" in interaction with a single "effect-part".

Axiom 1 (see [1] III, p. 202): The real function $\mu(\underline{\underline{V}}, \underline{\underline{F}})$ on $M^{\prime}$ fulfils the relation:

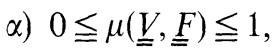

$\beta)$ for every $\underline{\underline{V}} \in \underline{\underline{K}}$ there is an $\underline{\underline{F}} \in \underline{\underline{L}}$ with $\mu(\underline{\underline{V}}, \underline{\underline{F}})=1$,

$\gamma$ ) there is a $\underline{\underline{F}} \in \underline{\underline{\underline{L}}}$ (denoted by 0 ), with $\mu(\underline{\underline{V}}, 0)=0$ for all $\underline{\underline{V}} \in \underline{\underline{K}}$.

The following axioms (see [1], p. 216, 217), play a significant role in the theory; in fact they imply the possibility of introducing the concept of a "microobject" as a "real" physical object into the theory (see [5], $\S 14$ and [6]).

Axiom 2a: The relation $\underline{\underline{V}}_{1} \widetilde{\underline{f}} \underline{\underline{V}}_{2}$ is an equivalence-relation;

Axiom $2 b:(f \times 1) \underline{\underline{M^{\prime}}}=\underline{\underline{K}} \times \underline{\underline{L}}$.

For the arguments in Ref. [1], Chapter III, it was essential to introduce (cf. p. 239 of [1]) the assumption (V 1). We will show below that some of the theorems deduced in Ref. [1] can be obtained without using (V 1).

In the mathematical literature the term "base-norm-space" is used for the type of spaces such as the space $B$ introduced in Ref. [1], Chapter III, $\S 3$. The case of a set $K$ "bounded below" (introduced in [1], Def. 3.10) is equivalent to the statement: $0 \notin K$. In this case, the cone $Q$ 
is usually called a "well based" cone. $0 \notin K$ holds because of Axiom $4 \mathrm{a}$ formulated below. By this axiom it follows that $K$ is a "base" of the cone $Q$. The assumption (V1) is called by mathematicians the "minimal decomposition property". The conventional mathematical term for $B^{\prime}$, the dual of the Banach space $B$, is the "order-norm-space" since the unit-ball of $B^{\prime}$ is the order-interval $[-1,1]$ (this result follows from Axiom 4a, cf. Ref. [1], Chapter III, Theorem 6.4).

Axiom $3 a, b$ (see [1], III, p. 243): The sets $\underline{\underline{K}}$ and $\underline{\underline{L}}$ are denumerable.

Axiom $4 a$ (see [1] III, p. 265): To every pair $F_{1} \in \bar{L}, F_{2} \in L$ and every number $\eta>0$ there exists a $F_{3} \in L$ with

a) $\mu\left(V, F_{1}\right)-\eta \leqq \mu\left(V, F_{3}\right)$ for all $V \in K$,

b) $\mu\left(V, F_{2}\right)-\eta \leqq \mu\left(V, F_{3}\right)$ for all $V \in K$,

c) $\mu\left(V, F_{3}\right)=0$ for all $V \in K_{0}\left(F_{1}\right) \cap K_{0}\left(F_{2}\right)$.

Without allowing any change in the physical interpretation of Axiom $4 b$ of Ref. [1], p. 277, it is possible to give a different formulation of this axiom which suggest more clearly its physical meaning:

Axiom $4 b$ : To every $F \in \hat{L}$ and every $\eta>0(\eta<1)$ there exists a number $\delta(\eta)>0(\delta(\eta)$ not depending on $F$ !) with $\delta(\eta) \rightarrow 0$ for $\eta \rightarrow 0$, and an $F^{\prime} \in L$ with $K_{0}\left(F^{\prime}\right) \supset K_{0}(F)$ and $\left[\mu\left(V, F^{\prime}\right) \geqq 1-\delta(\eta)\right.$ for all $V$ with $\mu(V, F) \geqq 1-\eta]$.

From this formulation of Axiom $4 \mathrm{~b}$ it follows immediately (because of $F^{\prime} \leqq E$ if $E \in G$ and $\left.K_{0}(E)=K_{0}(F)\right): \mu(V, E) \geqq 1-\delta(\eta)$ for all $V$ with $\mu(V, F) \geqq 1-\eta$. For all $V \in K_{1}(F)$ it follows (because of $\delta(\eta) \rightarrow 0$ for $\eta \rightarrow 0) \mu(V, E)=1$, i.e. $K_{1}(F) \subset K_{1}(E)$. Thus we recover the formulation of Axiom $4 \mathrm{~b}$ as given in [1], p. 277.

It is not difficult to give a physical interpretation of Axiom 5 of [1], p. 282, in terms of the "filters" $\underline{F} \in \underline{L}$ (see also [3]): If $C\left(V_{1}\right) \supset C\left(V_{2}\right)$ and $C\left(V_{1}\right) \neq C\left(V_{2}\right)$, so that $V_{1}$ has more "mixing-components" than $V_{2}$, then a filter-apparatus $\underline{F} \in \underline{L}$ can in principle be constructed such that it does not absorb to a physically measurable extent the objects of $V_{2}$, but still absorbs a physically measurable part of the ensemble $V_{1}$ : therefore we reformulate Axiom 5 of [1], p. 282, as follows:

Axiom 5. From $C\left(V_{1}\right) \supset C\left(V_{2}\right)$ and $C\left(V_{1}\right) \neq C\left(V_{2}\right)$ it follows: there is a number $\alpha>0$ so that for every number $\varepsilon>0$ an $\underline{F} \in \underline{L}$ exists, such that $\mu\left(V_{1}, \underline{F}\right)>0$ and $\mu\left(V_{2}, \underline{F}\right)<\varepsilon$.

The equivalence of this axiom to its formulation presented in [1], p. 282, can be seen in the following way: Using the formulation of Axiom 5 given above, it is easy to deduce (since $L$ is compact!).

Theorem 1.1. From $C\left(V_{1}\right) \supset C\left(V_{2}\right)$ and $C\left(V_{1}\right) \neq C\left(V_{2}\right)$ it follows: there exists an $F \in L$ with $\mu\left(V_{1}, F\right) \neq 0$ and $\mu\left(V_{2}, F\right)=0$.

This theorem is equivalent to each of the following theorems:

Theorem 1.2. $C\left(V_{1}\right) \supset C\left(V_{2}\right)$ and $C\left(V_{1}\right) \neq C\left(V_{2}\right)$ implies $L_{0}\left(V_{1}\right) \neq L_{0}\left(V_{2}\right)$. 
Theorem 1.3. $\left(V_{1} \stackrel{v}{=} V_{2}\right) \Rightarrow\left(V_{1} \stackrel{c}{=} V_{2}\right)$.

Theorem 1.3 however, corresponds to the formulation of Axiom 5 in [1], p. 282.

Proof of Theorem 1.3: If $C\left(V_{1}\right) \neq C\left(V_{2}\right)$ then $C\left(V_{1}\right)$ or $C\left(V_{2}\right)$ is a proper subset of $C\left(V_{1}, V_{2}\right)=C\left(V_{1}\right) \vee C\left(V_{2}\right)$. We can assume e.g. that $C\left(V_{1}, V_{2}\right)$ $\supset C\left(V_{2}\right)$ and $C\left(V_{1}, V_{2}\right) \neq C\left(V_{2}\right)$. If we put $C\left(V_{1}, V_{2}\right)=C\left(V_{3}\right)$ (see [1], III, Theorem 5.4) Theorem 1.2 gives $L_{0}\left(V_{3}\right) \neq L_{0}\left(V_{2}\right)$. Because of $L_{0}\left(V_{3}\right)$ $=L_{0}\left(V_{1}, V_{2}\right)=L_{0}\left(V_{1}\right) \cap L_{0}\left(V_{2}\right)$ it follows $L_{0}\left(V_{1}\right) \neq L_{0}\left(V_{2}\right)$ in contradiction to $V_{1} \stackrel{v}{=} V_{2}$.

\section{Some Consequences of the Axioms 1 to 5}

Theorem 2.1. $\hat{P}=\hat{\hat{L}}$ (thus we prove Theorem 8.9 of [1], III, $\S 8$, without using the assumption (V 1)!).

Proof. As shown in [1], p. 285-286, it is sufficient to prove, that for every $X_{0} \notin Q$ such that $\mu\left(X_{0}, 1\right)=1$ there exists an $F \in L$ such that $\mu\left(X_{0}, F\right)<0$.

By Theorems 4 and 5 of [7] there exists to every $X_{0}$ and every number $\varepsilon>0$ an element $F_{e} \in B^{\prime}$ with $0 \leqq F_{e} \leqq 1$ and $\left\|1-F_{e}\right\|<\varepsilon$, so that $X_{0}$ has a minimal decomposition relative to the base $K_{e}=Q \cap\left\{X \mid \mu\left(X, F_{e}\right)=1\right\}$; i.e. there is a decomposition

$$
X_{0}=\alpha X_{1}-\beta X_{2}
$$

with $X_{1}, X_{2} \in K_{e}$ and $\sup \left\{\left|\mu\left(X_{0}, Y\right)\right| \mid-F_{e} \leqq Y \leqq F_{e}\right\}=\alpha+\beta$. Since the set $-F_{e} \leqq Y \leqq F_{e}$ is $\sigma\left(B^{\prime}, B\right)$-compact, there exists a $Y_{0}$ with $\alpha+\beta=\mu\left(X_{0}, Y_{0}\right)$ $=\alpha \mu\left(X_{1}, Y_{0}\right)-\beta \mu\left(X_{2}, Y_{0}\right)$; this result implies (because of $\left|\mu\left(X, Y_{0}\right)\right| \leqq 1$ for all $\left.X \in K_{e}\right)$ the relations: $\mu\left(X_{1}, Y_{0}\right)=1$ and $\mu\left(X_{2}, Y_{0}\right)=-1$. Together with $X_{1}=\lambda_{1} V_{1}, X_{2}=\lambda_{2} V_{2}, V_{1} \in K, V_{2} \in K$ and $F_{0}=\frac{1}{2}\left(F_{e}-Y_{0}\right)$ one obtains the relations $\mu\left(V_{2}, F_{0}\right) \neq 0$ and $\mu\left(V_{1}, F_{0}\right)=0$ and $F_{0} \in \hat{P}$ (because of $\left.0 \leqq F_{0} \leqq F_{e} \leqq 1\right)$. Therefore we have $C\left(V_{1}\right) \neq C\left(V_{2}\right)$ and $C\left(V_{2}\right) \nsubseteq C\left(V_{1}\right)$. From Theorem 1.3 if follows, that there is an $F \in L$ with $\mu\left(V_{2}, F\right)>0$ and $\mu\left(V_{1}, F\right)=0$, so that

$$
\mu\left(X_{0}, F\right)=\alpha \mu\left(\lambda_{1} V_{1}, F\right)-\beta \mu\left(\lambda_{2} V_{2}, F\right)=-\beta \lambda_{2} \mu\left(V_{2}, F\right)<0 .
$$

Theorem 2.2. $\hat{L}=\hat{L}=\hat{P}$ (Thus we prove Theorem 8.11 of [1], III, $\S 8$, without using assumption (V 1)!).

Proof. The relation $\hat{L}=\hat{\hat{L}}=\hat{P}$ is proved, if, for every $X \in B$, with $\|X\|=1$ the relation

$$
\sup \{|\mu(X, 2 E-1)| \mid E \in G\}=1
$$

is valid, since then the convex and $\sigma\left(B^{\prime}, B\right)$-closed set spanned by the elements $(2 E-1)$ with $E \in G$ is the unit-ball of $B^{\prime}$. 
We use again the minimal decomposition, employed in the proof of Theorem 2.1:

$$
X=\alpha X_{1}-\beta X_{2}
$$

with $X_{1} \in K_{e}$ and $X_{2} \in K_{e}$.

If $X^{\prime} \in K_{e}$, than $\left\|X^{\prime}\right\|=\sup \mu\left(X^{\prime}, 1\right)$. From $\left\|1-F_{e}\right\| \leqq \varepsilon$ (where $\varepsilon$ can be chosen arbitrarily small) and

$$
\mu\left(X^{\prime}, F_{e}\right)=1 \text { for all } \quad X^{\prime} \in K_{e}
$$

it follows:

$$
\frac{1}{1+\varepsilon} \leqq\left\|X^{\prime}\right\| \leqq \frac{1}{1-\varepsilon} \text { for all } X^{\prime} \in K_{e} .
$$

Because of $0 \leqq F_{e} \leqq 1$ we have

$$
\alpha+\beta=\sup \left\{|\mu(X, Y)| \mid-F_{e} \leqq Y \leqq F_{e}\right\} \leqq\|X\| .
$$

On the other hand because of $X_{1}, X_{2} \in K_{e}$ the inequalities

$$
\|X\| \leqq \alpha\left\|X_{1}\right\|+\beta\left\|X_{2}\right\| \leqq(\alpha+\beta)(1-\varepsilon)^{-1}
$$

hold. Since we assumed $\|X\|=1$, we have

$$
1-\varepsilon \leqq \alpha+\beta \leqq 1 \text {. }
$$

The set $-F_{e} \leqq Y \leqq F_{e}$ being compact, there is a $Y_{0}$ such that $-F_{e} \leqq Y_{0}$ $\leqq F_{e}$ and

$$
\alpha+\beta=\mu\left(X, Y_{0}\right)=\alpha \mu\left(X_{1}, Y_{0}\right)-\beta \mu\left(X_{2}, Y_{0}\right) .
$$

This implies as in the proof of Theorem 2.1

$$
\mu\left(X_{1}, Y_{0}\right)=1 \text { and } \mu\left(X_{2}, Y_{0}\right)=-1 .
$$

Defining $F_{0}=\frac{1}{2}\left(Y_{0}+F_{e}\right)$ and putting $X_{1}=\left\|X_{1}\right\| V_{1}, X_{2}=\left\|X_{2}\right\| V_{2}$, $V_{1} \in K, V_{2} \in K$ we have $F_{0} \in \hat{P}=\hat{L}$,

$$
\left\|X_{1}\right\| \mu\left(V_{1}, F_{0}\right)=1 \text {, and } \mu\left(V_{2}, F_{0}\right)=0 .
$$

Because of $X_{1} \in K_{e}$ it follows

$$
\mu\left(V_{1}, F_{0}\right) \geqq 1-\varepsilon, \quad \mu\left(V_{2}, F_{0}\right)=0 .
$$

As follows from the above remarks (to Axiom $4 \mathrm{~b}$ ) we have the following result: for an $E \in G$ with $K_{0}\left(F_{0}\right)=K_{0}(E)$ there exists a number $\delta(\varepsilon)$ for which

$$
\mu(V, E) \geqq 1-\delta(\varepsilon) \quad \text { for all } V \text { with } \quad \mu(V, F) \geqq 1-\varepsilon .
$$


Therefore we have:

$$
\begin{aligned}
& \mu(X, 2 E-1)=2 \alpha\left\|X_{1}\right\| \mu\left(V_{1}, E\right)-\alpha\left\|X_{1}\right\| \\
& \quad-2 \beta\left\|X_{2}\right\| \mu\left(V_{2}, E\right)+\beta\left\|X_{2}\right\| \\
& =\alpha\left\|X_{1}\right\|\left[2 \mu\left(V_{1}, E\right)-1\right]+\beta\left\|X_{2}\right\| \geqq \frac{\alpha}{1+\alpha}(1-2 \delta(\varepsilon))+\frac{\beta}{1+\varepsilon} \\
& \geqq \frac{\alpha+\beta}{1+\varepsilon}-2 \delta(\varepsilon) \geqq \frac{1-\varepsilon}{1+\varepsilon}-2 \delta(\varepsilon) .
\end{aligned}
$$

Because of $\delta(\varepsilon) \rightarrow 0$ for $\varepsilon \rightarrow 0$, it follows $(\|X\|=1)$ :

$$
\sup \{|\mu(X, 2 E-1)| \mid E \in G\}=1 \text {. }
$$

Thus the theorem is proved. We note however, that without using assumption (V 1), we could not prove, that all exposed points of $\hat{P}=\hat{L}=\hat{L}$ are elements of $G$. Neither was it possible to prove without using assumption (V 1), that for every exposed point $F_{e}$ of $\hat{L}$ the sets $K_{1}\left(F_{e}\right)$ and $K_{0}\left(F_{e}\right)$ are not empty.

Theorem $9.2 \mathrm{~b}$ of [1], III, $\S 9$, can be reformulated as follows:

Theorem 2.3. If $B$ has the minimal-decomposition-property, i.e. if (V 1) is valid, the following relations $\alpha$ ) and $\beta$ ) are equivalent:

a) For every $E \in G$ with $E \neq 0$ the set $K_{1}(E)$ is not empty,

$\beta) G$ is the set of all exposed points of $\hat{L}=\hat{L}=\hat{P}$.

If (V 1) and one of the relations $\alpha$ ), $\beta$ ), are valid, then the following relations can be deduced (see [1], III, Theorem 1.4 and 9.5):

1) From $E_{1} \in G, E_{2} \in G$ and $E_{1} \leqq E_{2}$ follows that $E_{2}-E_{1} \in G$.

2) The mapping $E \rightarrow E^{*}=1-E$ of $G$ onto $G$ is an orthocomplementation of the lattice $G$. If the relation $\alpha$ ) of Theorem 2.3 is not valid, then the range of the mapping $E \rightarrow 1-E$ of $G$ into $\hat{L}$ is not necessarily equal to $G$. On the other hand because of $\hat{P}=\hat{\hat{L}}=\hat{L}$ the mapping $F \rightarrow 1-F$ is in involutory anti-automorphism of $\hat{L}$. Therefore the following theorem remains valid:

Theorem 2.4. The set of elements $E^{\prime} \in \hat{L}$ such that $E^{\prime}=1-E$ and $E \in G$, forms a lattice $G^{\prime} . E^{\prime}$ is the smallest element of $L_{1} K_{1}\left(E^{\prime}\right)$. The mapping $E \rightarrow 1-E$ is a dual isomorphism of $G$ onto $G^{\prime}$.

Theorem 2.5. The following relations $\alpha$ ), $\beta$ ) and $\delta$ ) are equivalent and $\beta$ ) implies $\gamma$ ) (the assumption (V1) is not necessary for the proof):

ג) $G=G^{\prime}$,

$\beta)$ For every $E \in G, E \neq 0$ the set $K_{1}(E)$ is not empty.

$\gamma)$ all elements of $G$ are exposed points of $\hat{L}=\hat{L}=\hat{P}$,

ס) From $E_{1} \in G, E_{2} \in G$ and $E_{1} \leqq E_{2}$ it follows $E_{2}-E_{1} \in G$. 
Proof. Putting $E_{2}=1$, we get $\left.\delta\right) \Rightarrow \alpha$ ).

Since $K_{1}(E)=K_{0}(1-E), K_{1}(E)$ is not empty, if $1-E \neq 1$, i.e. if $E \neq 0$; thus we have $\alpha) \Rightarrow \beta$ ).

$\beta) \Rightarrow \delta$ ) is proved in [1], III, Theorem 9.4.

$\beta) \Rightarrow \gamma)$ is proved in [1], p. 294.

From Theorem 2.5 we see that all theorems in Ref. [1], Chapter III, concerning the lattice $G$ can be deduced from Axiom $4 \mathrm{bz}$ without using (V 1). By Theorem 2.5, Axiom $4 \mathrm{bz}$, is equivalent to the following condition: $E \rightarrow 1-E$ is an orthocomplementation of the lattice $G$. In a general case (i.e. without assuming the validity of Axiom $4 \mathrm{bz}$ ) one also could consider the possibility $G \neq G^{\prime}$. However, in that case, the map $E \rightarrow 1-E$ is a dual isomorphism of $G$ onto $G^{\prime}$. We then have: $\overline{\mathrm{co}} G$ $=\overline{\mathrm{CO}} G^{\prime}=\hat{P}=\hat{\hat{L}}=\hat{L}$ (the symbol $\overline{\mathrm{co}}$ denoting the $\sigma\left(B^{\prime}, B\right)$ closed convex hull). Therefore the $\sigma\left(B^{\prime}, B\right)$-closure $\bar{G}$ of $G$ contains all extremal points of $\hat{L}=\hat{\hat{L}}=\hat{P}$.

The conjecture that perhaps the case $G \neq G^{\prime}$ could be of significance in a physical theory cannot be a priori rejected. However, one important point should be emphasized here:

The case $G \neq G^{\prime}$ cannot be distinguished from the case $G=G^{\prime}$ on the basic of physical experiments only, since Axiom $4 \mathrm{bz}$ has no direct physical interpretation. Axiom 4 bz is only a mathematical "idealization". Therefore the case $G \neq G^{\prime}$ could only be of interest if other mathematical idealizations introduced into the theory might contradict Axiom $4 \mathrm{bz}$, i.e. the relation $G=G^{\prime}$. If, in theoretical physics, one encounters mathematical inconsistencies, i.e. self-contradictory mathematical theories then one may suspect that two incompatible mathematical idealizations could have been introduced into the theory. The "current" quantum field theory seems to be an example of such a situation the search for a consistent mathematical formulation of the field theory being a night-mare of many a physicist.

By Theorem 2.5, the significance of the "idealization" implied by Axiom $4 \mathrm{bz}$ also becomes clear. We regret that it was not possible to find a similar theorem clearing up the significance of assumption (V 1).

Using Theorem 8.10 of Ref. [1], Chapter III, we only can prove the following:

Theorem 2.6. From (V 1) (without using Axiom 4bz) it follows that $G$ contains all exposed points of $\hat{P}=\hat{L}=\hat{L}$.

One aspect of the significance of the assumption (V1) and the Axiom $4 \mathrm{bz}$ is suggested by Theorem 2.3. At present we are not able to formulate in terms of $G$ and $\hat{P}=\hat{L}=\hat{L}$ any necessary and sufficient conditions for the validity of (V 1). 


\section{The General Covering Condition}

Let us assume the validity of Axiom $4 \mathrm{bz}$ without assuming the validity of (V 1). Then all the Theorems 9.5 to 9.11 of Ref. [1], Chapter III, can be proved. The physical interpretation of Axiom 6 of Ref. [1], Chapter III, $\S 1$, as given in Ref. [2] is an improvement of the formulation of $\S 10$ of Chapter III, Ref. [1] and does not depend on the assumption (V 1). In [2] we tried to demonstrate on some examples of physical experiments, how one is lead to the following two conditions:

a) $\inf \left\{\mu\left(V, E_{1}\right) \mid V \in C\left(\frac{1}{2} V_{2}+\frac{1}{2} V_{4}\right)\right\} \neq 0$ (with $E_{1} \in G$ and $C\left(V_{1}\right)=K_{0}\left(E_{1}\right)$ ) and $V_{4} \perp V_{2}$ implies the existence of an $F \in L_{0}\left(V_{1}\right)$ with $\mu\left(V_{2}, F\right)=0$ and $\mu\left(V_{4}, F\right) \neq 0$.

b) From inf $\left\{\mu\left(V, E_{24}\right)\left\{V \in C\left(V_{1}\right)\right\} \neq 0\right.$ (with $E_{24} \in G$ and $C\left(\frac{1}{2} V_{2}+\frac{1}{2} V_{4}\right)$ $\left.=K_{0}\left(E_{24}\right)\right)$ and $V_{4} \perp V_{2}$ it follows, that for $E_{4} \in G$ with $C\left(V_{4}\right)=K_{1}\left(E_{4}\right)$ the relation $\mu\left(V, E_{4}\right) \neq 1$ is valid for all $V \in C\left(\frac{1}{2} V_{1}+\frac{1}{2} V_{2}\right)$.

The two conditions a) and b) are equivalent - as shown in [2] - to the "general covering condition", i.e. to

Axiom 6: $C\left(V_{1}\right) \subset C\left(V_{3}\right) \subset C\left(\frac{1}{2} V_{1}+\frac{1}{2} V_{2}\right)$ and $\Delta\left(C\left(V_{3}\right), C\left(V_{2}\right)\right) \neq 0$ implies $C\left(V_{3}\right)=C\left(V_{1}\right)$.

The expression $\Delta(\cdot, \cdot)$ in Axiom 6 is defined by the equation:

$$
\begin{aligned}
\Delta\left(C\left(V_{1}\right), C\left(V_{2}\right)\right)= & \frac{1}{2}\left[\inf \left\{\mu\left(V, E_{1}\right) \mid V \in C\left(V_{2}\right)\right\}\right. \\
& \left.+\inf \left\{\mu\left(V, E_{2}\right) \mid V \in C\left(V_{1}\right)\right\}\right],
\end{aligned}
$$

where the two elements $E_{1}$ and $E_{2}$ of $G$, are defined by the equations:

$$
C\left(V_{1}\right)=K_{0}\left(E_{1}\right) \text { and } C\left(V_{2}\right)=K_{0}\left(E_{2}\right) .
$$

It is interesting to note that one does not need the assumption (V 1) to deduce the representation of the elements of $K$ and $L$ by operators in a Hilbert space if the assumption (V 3) is valid (cf. [1], p. 415). Once the representation of the elements of $K$ and $L$ by operators in a Hilbert space has been proved, the relation (V 1) follows easily. The representation theorem is proved in Ref. [8] using the results of Refs., [2] and [1], Chapter III, § 18 .

The assumption (V 1) does not seem to be so important as orginally supposed in [1]. Only the idealization implied by Axiom 4 bz seems to play an essential role in the theory.

\section{References}

1. Ludwig, G.: Deutung des Begriffs „physikalische Theorie“ und axiomatische Grundlegung der Hilbertraumstruktur der Quantenmechanik durch Hauptsätze des Messens. Lecture Notes in Physics 4. Berlin-Heidelberg-New York: Springer 1970.

2. - A physical interpretation of an axiom within an axiomatic approach to quantum mechanics and a new formulation of this axiom as a general covering condition. Notes in Math. Phys. 1. Marburg (1971). 
3. - The measuring progress and an axiomatic foundation of quantum mechanics. Notes in Math. Phys. 3. Marburg (1971).

4. - Transformationen von Gesamtheiten und Effekten. Notes in Math. Phys. 4. Marburg.

5. - Meß- und Präparierprozesse. Notes in Math. Phys. 6. Marburg (in preparation).

6. - Das Problem der Wirklichkeit der Mikroobjekte. Notes in Math. Phys. 8, Marburg (in preparation).

7. Ellis, A. J.: Minimal decompositions in partially ordered normed vector spaces. Proc. Cambridge Phil. Soc. 64, 989-1000 (1968).

8. Stolz, P.: Attempt of an axiomatic foundation of quantum mechanics and more general theories. VI. Commun. math. Phys. 23, 117-126 (1971).

G. Ludwig

Institut für Theoretische Physik (I)

der Universität

D-3550 Marburg, Renthof 7

Germany 